\title{
RISK-BASED MAINTENANCE ASSESSMENT IN THE MANUFACTURING INDUSTRY: MINIMISATION OF SUBOPTIMAL PRIORITISATION
}

\author{
R.M. Chandima Ratnayake ${ }^{1}$, Katarzyna Antosz ${ }^{2}$ \\ ${ }^{1}$ University of Stavanger, Faculty of Science and Technology, Department of Mechanical and Structural Engineering \\ and Materials Science, Norway \\ ${ }^{2}$ Rzeszow University of Technology, Faculty of Mechanical Engineering and Aeronautics, Poland
}

Corresponding author:

Katarzyna Antosz

Rzeszow University of Technology

Department of Manufacturing and Production Engineering

Al. Powstancow Warszawy 8, 35-959 Rzeszow, Poland

phone: (+4817) 865-14-52

e-mail: katarzyna.antosz@prz.edu.pl

Received: 10 October 2016

Accepted: 30 January 2017

\begin{abstract}
Manufacturing firms continuously strive to increase the efficiency and effectiveness in the maintenance management processes. Focus is placed on eliminating the unexpected failures which cause unnecessary costs and the production losses. Risk-based maintenance (RBM) strategies enable to address the above through the identification of probability and consequences of potential failures whilst providing a way for prioritisation of maintenance actions based on the risk of possible failures. Such prioritisations enable to identify the optimal maintenance strategy, intervals of maintenance tasks, and optimal level of spare parts inventory. However, the risk assessment activities are performed with the support of a risk matrix. Suboptimal classifications and/or prioritisations arise due to the inherent nature of the risk matrix. This is caused by the fact that there are no means to incorporate actual circumstances at the boundary of the input ranges or at the levels of linguistic data and risk categories. In this paper, a risk matrix is first developed in collaboration with one of the manufacturing firms in Poland. Then, it illustrates the use of fuzzy logic for minimisation of suboptimal prioritisation and/or classifications using a fuzzy inference system (FIS) together with illustrative membership functions and a rule base. Finally, an illustrative risk assessment is also demonstrated to illustrate the methodology.
\end{abstract}

KEYWORDS

classification, fuzzy logic, manufacturing firms, risk based maintenance, risk matrix.

\section{Introduction}

The performance of production/manufacturing firms is heavily influenced by the maintenance productivity [1] which concerns significant tasks that deal with inspections, scheduled cleaning, adjustments, repairs, and replacements of machinery to ensure operational reliability and the final product quality $[2-5]$. The output of a manufacturing process is dependent on the performance of machinery as defective products from the previous machinery can accumulate or disturb the subsequent process and overall quality. This is further exacerbated by the increasing trend of mechanisation and automation. The role of machinery in production operations becomes a significantly important factor. Hence, it is vital to keep the machinery in an ideal condition and operating effectively [6].

Naturally, the equipment or machinery gets older and deteriorates with time and/or with the level of usage in a manufacturing process, which has a direct/indirect impact on the overall quality of the manufactured products. In this context, it is possible to characterise the diminished product quality by the increasing rejection rate and declining performance of a particular piece of machinery. As the 
rejected (or non-conformed) products cause the deterioration of the downstream process, it is not possible to separate the maintenance tasks of machinery from the tasks of the overall management of the manufacturing process[5]. Moreover, performing the maintenance at the right time on the right machinery by the right personnel is crucial to restore it to an acceptable condition. Hence, it is vital to use effective approaches to machinery prioritisation to schedule maintenance tasks and assign them with different maintenance strategies (i.e. preventive or corrective) as appropriately based on the risk [i.e. risk-based maintenance (RBM)] of the potential failures [7]. Currently, some of the MFs (Manufacturing Firms) use empirical models to classify the machinery for performing maintenance tasks $[8,9]$. However, it is known that empirical models based on classification require further fine prioritisation of the machinery for the allocation of existing resources for performing maintenance tasks. Therefore, it is vital to develop the RMB approach for prioritisation and classification of machinery for effective scheduling of maintenance tasks. First, this work explains the conditions of the problem of the currently existing empirical model-based approach. Then, it suggests a risk matrix to perform the machinery prioritisation and classification by considering personal safety $(\mathrm{PS})$, the percentage of non-conforming products (PNCP), availability (A) of the machinery for manufacturing tasks a month, and the Overall Equipment Efficiency OEE a month. Finally, it demonstrates a fuzzy logic-based approach that supports the use of a risk matrix to make the optimal prioritisation and classification of the machinery (i.e. how fuzzy logic enables to minimise sub-optimal classifications when the RBM scheduling is made with the support of a risk matrix).

\section{Case study and industrial challenge}

The case-study manufacturing firm (MF) is located in the Podkarpackie voivodeship (Poland). This company produces plastic car parts for the automotive industry such as: coolant tanks, brake fluid tanks, washer fluid tanks and various covers, housings, handles, plugs, sensors, etc. These parts are used in many car brands around the world. The main types of machines used in the production process are injection moulding machines and sealing devices. The studied company does not apply the same maintenance strategy for all its machines. The type of a maintenance strategy depends on the type of a machine. For the new machines, the company realises maintenance activities under warranty. For other machines, the company partially implements preventive maintenance (PM) and condition-based maintenance (CBM). At the end of each manufacturing year, a maintenance supervisor and a production manager are responsible for preparing a schedule for the preventive maintenance tasks. Depending on the level of criticality, machines, in general, must have at least one inspection completed a year. The maintenance schedule is updated once a year, considering unexpected failures of the previous year as well as the value of MTBF (the Mean Time Between Failures) to increase or decrease the frequency of inspections. Additionally, for the machines which had the largest number of unexpected failures in the previous year or a very low value of MTBF, the maintenance supervisor plans a special control. This special control involves constant monitoring of the technical condition of the machine. The CMMS (computer-aided maintenance management system) helps the company with gathering and analysing the information of the machines and production. The CMMS is also used to generate the values of indicators (i.e. OEE, MTBF) and graphs, etc. to support/schedule maintenance tasks. The supervision of machines is carried out via the inspection of the individual machine components by technical staff in the maintenance department.

Even though the MF uses maintenance strategies mentioned before, it still has to face numerous challenges. For instance, as the MF has insufficient personnel in the maintenance department, it is not possible to perform maintenance tasks according to the schedule. The increase of maintenance backlog is also caused by a higher number of client orders resulting in no possibility to stop the machine and perform inspection tasks according to the maintenance schedule, or other routine activities to identify the real technical condition of the machine. On the other hand, to identify the real technical condition of a machine, it is essential for the technical staff to accommodate themselves with the specialised equipment and devices (e.g. for the measurement of temperature and vibration of machine components). As this equipment and devices are very expensive, the maintenance department has a limited amount to perform the maintenance tasks effectively. Hence, it has been revealed that currently, the MF experiences a significantly large number of breakdowns resulting in a significantly low MTBF indicator for machines. It means that the MF must perform, from time to time, the unplanned corrective or reactive maintenance (i.e. firefighting). In addition, the MF has not established the rules concerning time limits and the way to control the efficiency of machines (i.e. a monitoring task has been performed in an un- 
planned manner). Hence, to change the current situation, the MF needs to change the currently used maintenance assessment approaches and strategies. The MF is in the process of changing the current maintenance strategies into an RBM strategy. Hence, it is vital to develop a risk matrix and an approach to make risk-based machinery classification for deploying maintenance resources effectively. As the first step, the case-study MF implemented the machine classification criteria. The main goal of the machines classification is to identify the machines which need special control. The used classification model is based on the four criteria: the production process $(\mathrm{PP})$, failures (F), quality (Q), and employee safety (S). Table 1 illustrates the machine classification criteria and the description of each criterion.

The first criterion describes the work time of a machine in a production process. The scale for this criterion ranges from 1 to 4 points. The next criterion for the machine classification is failures. This criterion is assessed by machine interchangeability in the case of failure, the cost of failure elimination, and the number of failures per year. The scale for each subcriterion ranged from 1 to 3 points. Another criterion in this classification is the quality. In this criterion, the company uses the percentage of nonconforming products per year. The last criterion is the employee safety. Scale scores for the quality and safety range from 1 to 3 points. For the safety criterion, 1 point is assigned to the machines whose failure will have a major impact on the health of the worker, and 3 points are given to those whose failures have a minor risk to the workers' health.

The $C_{m}$ values are calculated using the empirical formula (1) to qualify a machine for a certain classification category.

$$
C_{m}=w_{i} P_{w}+w_{i}\left(I_{f}+C_{f}+N_{f}\right)+w_{i} N_{q}+w_{i} S_{p} .
$$

All parameters $P_{w}, I_{f}, C_{f}, N_{f}, N_{q}, S_{p}$ and the weights $w_{i}$ shall be decided by the management of a particular MF based on their requirements.

Formula (2)

$$
\sum w_{i}=1
$$

Based on the discussion carried out with the people responsible for performing maintenance activities in the MF, weights $\left(w_{i}\right)$ of the formula (1) were decided. The formula (3) illustrates the selected values of weights

$$
\begin{aligned}
C_{m}= & 0.3 P_{w}+0.2\left(I_{f}+C_{f}+N_{f}\right) \\
& +0.25 N_{q}+0.25 S_{p} .
\end{aligned}
$$

\begin{tabular}{|c|c|c|c|c|}
\hline Classification criteria & Description & Symbol & Alternatives & Points \\
\hline \multirow{4}{*}{ Production process (PP) } & \multirow{4}{*}{ Work time of machine } & \multirow{4}{*}{$P_{w}$} & 3 shifts & 4 \\
\hline & & & 2 shifts & 3 \\
\hline & & & 1 shift & 2 \\
\hline & & & Occasionally & 1 \\
\hline \multirow{9}{*}{ Failures (F) } & \multirow{3}{*}{$\begin{array}{l}\text { Machine interchangeability } \\
\text { in case of failure }\end{array}$} & \multirow{3}{*}{$I_{f}$} & $\begin{array}{l}\text { No possibility to move a production process } \\
\text { to another work-stand }\end{array}$ & 3 \\
\hline & & & $\begin{array}{c}\text { It is possible to move a production process } \\
\text { to another workstand but production } \\
\text { of another production process } \\
\text { has to be stopped }\end{array}$ & 2 \\
\hline & & & $\begin{array}{c}\text { It is possible to move a production process } \\
\text { to another workstand } \\
\text { (there are few machines) }\end{array}$ & 1 \\
\hline & \multirow{3}{*}{ Cost of failures elimination } & \multirow{3}{*}{$C_{f}$} & High & 3 \\
\hline & & & Medium & 2 \\
\hline & & & Low & 1 \\
\hline & \multirow{3}{*}{ Number of failures per year } & \multirow{3}{*}{$N_{f}$} & More than 5 per year & 3 \\
\hline & & & $3-5$ per year & 2 \\
\hline & & & Less than 3 per year & 1 \\
\hline \multirow{3}{*}{ Quality (Q) } & \multirow{3}{*}{$\begin{array}{l}\text { Percentage of nonconforming } \\
\text { products per year [\%] }\end{array}$} & \multirow{3}{*}{$N_{q}$} & More than $5 \%$ & 3 \\
\hline & & & $3-5 \%$ & 2 \\
\hline & & & Less than $3 \%$ & 1 \\
\hline \multirow{3}{*}{ Safety (S) } & \multirow{3}{*}{ Personnel safety } & \multirow{3}{*}{$S_{p}$} & Fatality & 1 \\
\hline & & & Serious personnel injury & 2 \\
\hline & & & First aid & 3 \\
\hline
\end{tabular}

Table 1

Criteria of machine classification in the company. 
The classification model classifies all machines into three categories of importance in the maintenance process: $\mathrm{H}$ - High, $\mathrm{M}$ - Medium and L - Low. Table 2 shows the machine classification categories according to the company model.

Table 2

Machine classification categories.

\begin{tabular}{c|c|c|c}
\hline $\begin{array}{c}\text { Classification } \\
\text { categories }\end{array}$ & $\begin{array}{c}\text { Category } \\
\text { I-H }\end{array}$ & $\begin{array}{c}\text { Category } \\
\text { II-M }\end{array}$ & $\begin{array}{c}\text { Category } \\
\text { III-L }\end{array}$ \\
\hline Range of $C_{m}$ & $\geq 2.5$ & $2.5-1.5$ & $\leq 1.4$ \\
\hline Value & $30 \%$ & $25 \%$ & $45 \%$ \\
\hline
\end{tabular}

All the machines were classified. As a result, $30 \%$ were assigned to the Category I (priority $\mathrm{H}$ ); $25 \%$ were assigned to the Category II (priority M), and $45 \%$ were assigned to the Category III (priority L). In this context, it is obviously necessary to pay special attention to the machines that come under the Category I (priority H).

In practice, for the MF, it is a difficult task to treat $30 \%$ of the machines as the priority $\mathrm{H}$, as it is a medium size MF with no sufficient resources such as personnel and equipment. Therefore, to allocate the available resources effectively for the maintenance tasks, it is vital to perform the RBM prioritisation of the existing machinery. Hence, it is es- sential to develop a risk matrix together with an effective risk analysis process to optimise the maintenance resources allocation. The risk matrix was developed based on the authors' experience, historical data from the case study manufacturing firm, and with the support of a maintenance supervisor.

Table 3 shows ranges, ranks, and linguistic terms for the consequences and failure frequency. Three types of consequences are defined: personal safety (PS), the percentage of non-conforming products (PoNCP), availability (A), and the machinery failure frequency [i.e. frequency of failure $(\mathrm{FoF})$ ] that is designated as the mean time between failures (MTBF).

The ranges and ranks of input and output variables are used as the basis for input and output variables. These ranges and rules are developed by means of the information available in the CMMS system. Then, based on the risk of potential loss of quality (i.e. MTBF of machinery vs. PoNCP), personnel safety (i.e. MTBF vs. PS) and production (i.e. MTBF vs. A), the machinery was evaluated with the focus on classifying them into groups (i.e. risk-based prioritisation of machinery) for performing the right maintenance strategy - maintenance tasks. Table 4 illustrates the developed risk matrix and possible risk scenarios.

Table 3

Ranges, ranks, and linguistic terms for consequences and the number of failures.

\begin{tabular}{|c|c|c|c|c|c|c|}
\hline \multirow{7}{*}{ Input variable } & & \multicolumn{5}{|c|}{ Consequences } \\
\hline & Factors & \multicolumn{5}{|c|}{ Levels of consequence due to a functional failure } \\
\hline & Rank & 1 & 2 & 3 & 4 & 5 \\
\hline & Linguistic term & Very high & High & Medium & Low & Very low \\
\hline & $\mathrm{PS}$ & Fatality & Permanent injury & Serious personnel injury & Medical treatment & First aid \\
\hline & PoNCP & $>5 \%$ & $(3-5) \%$ & $(1.5-3) \%$ & $(0.5-1.5) \%$ & $<0.5 \%$ \\
\hline & $\mathrm{A}(\%)$ & $<20$ & 20 to 40 & 40 to 60 & 60 to 80 & 80 to 100 \\
\hline \multirow{3}{*}{$\begin{array}{c}\text { Frequency } \\
\text { of failure }(\mathrm{FoF})\end{array}$} & Rank & 1 & 2 & 3 & 4 & 5 \\
\hline & Linguistic term & Very high & High & Medium & Low & Very low \\
\hline & MTBF (hours) & Less than 200 & $200-400$ & $400-600$ & $600-800$ & More than 800 \\
\hline
\end{tabular}

Table 4

Risk matrix: overall risk scenarios.

\begin{tabular}{|c|c|c|c|c|c|c|c|c|}
\hline & & & & & & Jonsequences & & \\
\hline & & & Linguistic term & $\mathrm{VH}$ & $\mathrm{H}$ & $\mathrm{M}$ & $\mathrm{L}$ & VL \\
\hline Lin & guistic & Consequences & $\mathrm{PS}$ & Fatality & Permanent injury & Serious personnel injury & Medical treatment & First aid \\
\hline & erm & & PoNCP & $>5 \%$ & $3-5 \%$ & $1.5-3 \%$ & $1.5-0.5 \%$ & $<0.5 \%$ \\
\hline & & & $\mathrm{A}(\%)$ & $<20$ & 20 to 40 & 40 to 60 & 60 to 80 & 80 to 100 \\
\hline & & MTBF (hours) & Rank & 1 & 2 & 3 & 4 & 5 \\
\hline & $\mathrm{VH}$ & Less than 200 & 1 & $\mathrm{VH}$ & $\mathrm{VH}$ & $\mathrm{VH}$ & $\mathrm{H}$ & $\mathrm{M}-\mathrm{H}$ \\
\hline & $\mathrm{H}$ & $200-400$ & 2 & $\mathrm{VH}$ & $\mathrm{VH}$ & $\mathrm{H}$ & $\mathrm{M}-\mathrm{H}$ & $\mathrm{M}-\mathrm{H}$ \\
\hline FoF & $\mathrm{M}$ & $400-600$ & 3 & $\mathrm{VH}$ & $\mathrm{H}$ & $\mathrm{M}-\mathrm{H}$ & M-L & M-L \\
\hline & $\mathrm{L}$ & $600-800$ & 4 & $\mathrm{VH}$ & $\mathrm{M}-\mathrm{H}$ & M-L & $\mathrm{L}$ & $\mathrm{L}$ \\
\hline & $\mathrm{VL}$ & More than 800 & 5 & $\mathrm{VH}$ & $\mathrm{M}-\mathrm{H}$ & M-L & $\mathrm{L}$ & $\mathrm{VL}$ \\
\hline
\end{tabular}

VH - Very high; H - High; M-H - Medium to high; M-L - Medium to low; L - Low; VL - Very low 
The potential use of the risk matrix was verified together with the maintenance personnel in the studied MF. If the values of both, the number of failures and the consequences, are in the middle of each range, then there is no challenge in estimating the level of risk. However, if a range value falls to the border of a particular range, then there is a high possibility of making suboptimal classification of the machinery during the risk-based maintenance prioritisations. In addition, such uncertainty creates significant variability in the analysis depending on the available information, knowledge, and experience. To cater these circumstances, it is vital to use a fuzzy logic-based approach for enhancing the RBM classification of the machinery. In this context, it is possible to use the risk matrix as the rule base for a fuzzy inference system.

\section{Fuzzy logic assisted RBM assessment approach}

The illustrative case presented in this work utilises a Mamdani-type fuzzy inference process [10]. The membership functions are developed with consideration to the ranges of MTBF and CoFs. However, it is possible to develop membership functions with the support of maintenance experts by examining how they perceive membership values along the ranges, Stadnicka et al. [11]. Figure 1 illustrates an overall view of the proposed fuzzy risk rank assessment system. It is possible to select MTBF and $\mathrm{CoF}$ as the input to the fuzzy inference system to calculate the risk rank.

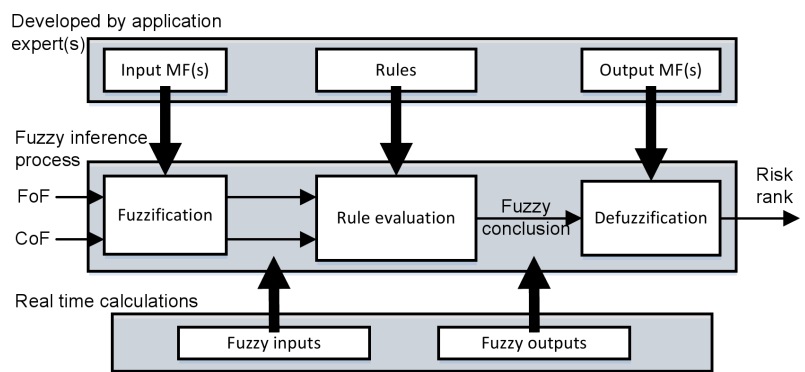

Fig. 1. Overall view of the fuzzy RBM assessment process.

The illustrative case was demonstrated by means of triangular and trapezoidal membership functions. Fuzzy logic assisted risk rank calculation was demonstrated with MTBF and the consequence of failure with PoNCP.
The input and output variables consist of quantitative, qualitative, and judgmental (i.e. linguistic) data. Using an appropriate membership function, the user has 'more confidence' that the input parameter lies in the centre of the interval than at the edges. In this study, the authors incorporated Gaussian membership functions, such as in works by Tay and Lee [12], Ratnayake [13], Hameed [14], in order to minimise the gap between practical realities and mathematical modelling, which are defined by Eq. (4):

$$
\operatorname{Gaussian}(x ; c, \sigma)=e^{\frac{-(x-c)^{2}}{2 \sigma^{2}}},
$$

where $c$ represents the centre and $\sigma$ determines the width of the MFs. To model the membership functions, the Gaussian combination membership (GCMF) (i.e. 'gauss2mf'), which is available in MATLAB (R2014b), was used [15, 16]. The function 'gauss2mf' is a combination of two parameters [i.e. $(c, \sigma)]$ indicated in Eq. (5). It follows the syntax from Mathworks [15]:

$$
y=\operatorname{gauss} 2 \operatorname{mf}\left\{x\left[\begin{array}{llll}
\sigma_{1} & c_{z} & \sigma_{2} & c_{2}
\end{array}\right]\right\} .
$$

The first part of the function of the GCMF is specified by $\sigma_{1}$ and $c_{1}$, which determine the shape of the left-most curve. The second part of the GCMF, specified by $\sigma_{2}$ and $c_{2}$, determines the shape of the right-most curve. Whenever $c_{1}<c_{2}$, the 'gauss2mf' function reaches a maximum value of 1 . Otherwise, the maximum value is less than one. The order of the parameters is as follows: $\left[\begin{array}{llll}\sigma_{1} & c_{1} & \sigma_{2} & c_{2}\end{array}\right]$ [13]. Moreover, other parameters of the fuzzy logic-based expert system that had been selected for the current analysis are as follows: 'AND' operator with 'minimum', 'OR' operator with 'maximum', 'Implication' with 'minimum', 'Aggregation' with 'maximum' and 'Defuzzification' with 'centroid' algorithm. A fuzzy rule base was developed with a table-look-up approach (Table 4). The toolbox simulator tool of MATLAB (R2014b) was used to execute the suggested fuzzy inference process in Matlab program [16].

\section{Analysis, results, and discussion}

Figure 2 illustrates Matlab R2014b based fuzzy logic designer used for calculating risk ranks in relation to MTBF and PoNCP. The parameter values of GCMFs are presented in Table 5 .

Using the risk matrix (Table 4), a rule base was developed (Fig. 3).

The GCMFs for the PoNCP, MTBF, and Risk rank are illustrated in Figs. 4-6, respectively. 
Table 5

Gaussian MF parameters for input and output variables.

\begin{tabular}{|c|c|c|c|c|c|c|}
\hline Input variable & $\mathrm{VH}$ & $\mathrm{H}$ & $\mathrm{M}$ & \multicolumn{2}{|c|}{$\mathrm{L}$} & $\mathrm{VL}$ \\
\hline MTBF & $\left.\begin{array}{lllll}40 & 100 & 40 & 200\end{array}\right]$ & {$\left[\begin{array}{lllll}40 & 300 & 65 & 300\end{array}\right]$} & $\left.\begin{array}{lllll}80 & 500 & 80 & 500\end{array}\right]$ & \multicolumn{2}{|c|}{$\left[\begin{array}{lllll}65 & 700 & 40 & 700\end{array}\right]$} & {$\left[\begin{array}{lllll}40 & 800 & 40 & 900\end{array}\right]$} \\
\hline PoNCP & {$\left[\begin{array}{llll}0.4 & 5 & 0.5 & 5\end{array}\right]$} & {$\left[\begin{array}{llll}0.6 & 4 & 0.3 & 4\end{array}\right]$} & {$\left[\begin{array}{lllll}0.4 & 2.25 & 0.6 & 2.25\end{array}\right]$} & \multicolumn{2}{|c|}{$\left[\begin{array}{llll}0.2 & 1 & 0.5 & 1\end{array}\right]$} & {$\left[\begin{array}{llll}0.2 & 0 & 0.2 & 0.5\end{array}\right]$} \\
\hline Output variable & VH & $\mathrm{H}$ & $\mathrm{M}-\mathrm{H}$ & M-L & $\mathrm{L}$ & VL \\
\hline Risk rank & {$\left[\begin{array}{lllll}0.2 & 0 & 0.2 & 0.5\end{array}\right]$} & {$\left[\begin{array}{ll}0.3 & 1\end{array}\right]$} & {$\left[\begin{array}{ll}0.3 & 2\end{array}\right]$} & {$\left[\begin{array}{ll}0.3 & 3\end{array}\right]$} & {$\left[\begin{array}{lll}0.3 & 3.75\end{array}\right]$} & {$\left[\begin{array}{lllll}0.2 & 4.5 & 0.1 & 5\end{array}\right]$} \\
\hline
\end{tabular}

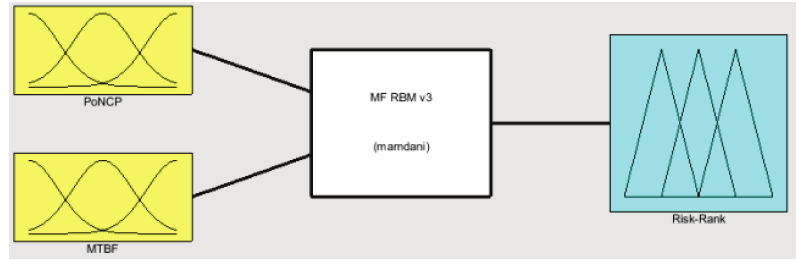

Fig. 2. Fuzzy logic designer.

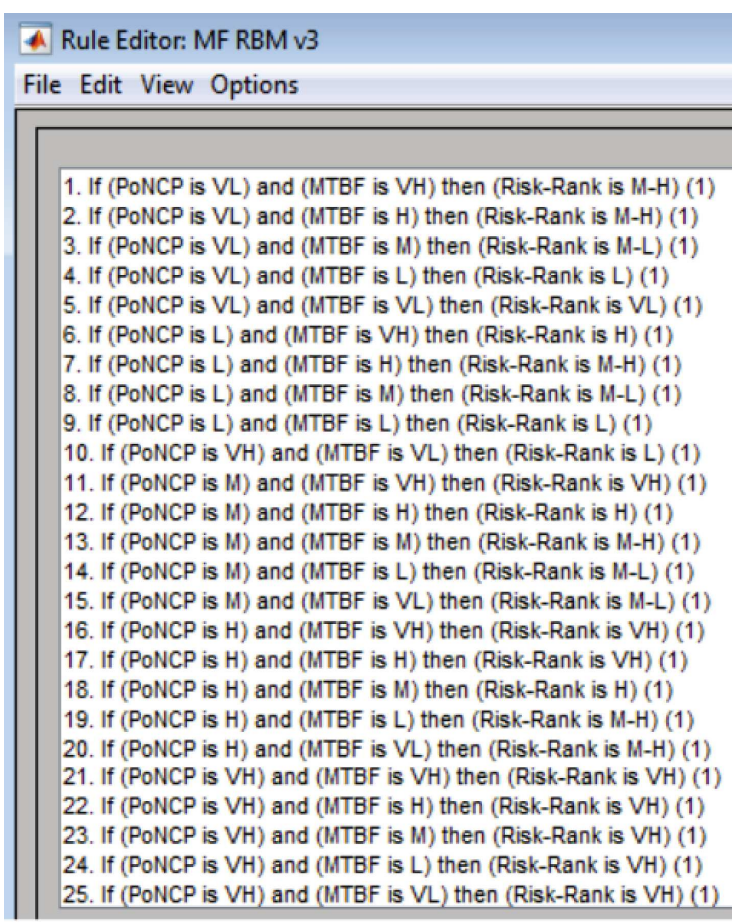

Fig. 3. Rule base.

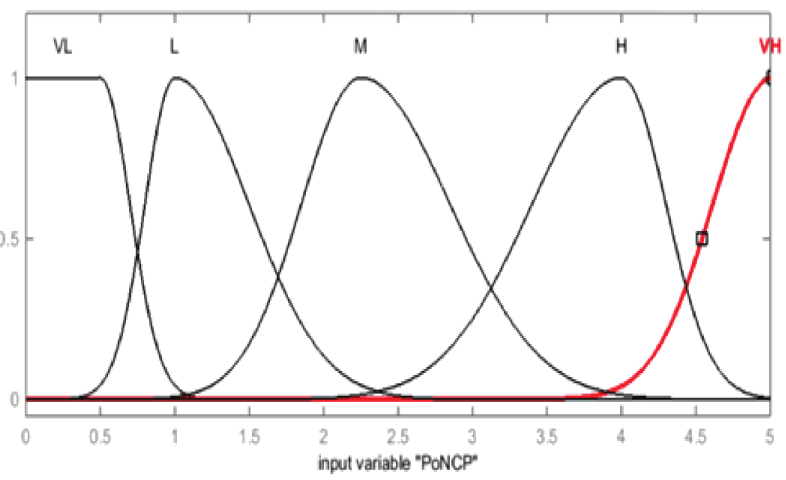

Fig. 4. GCMF of PoNCP.

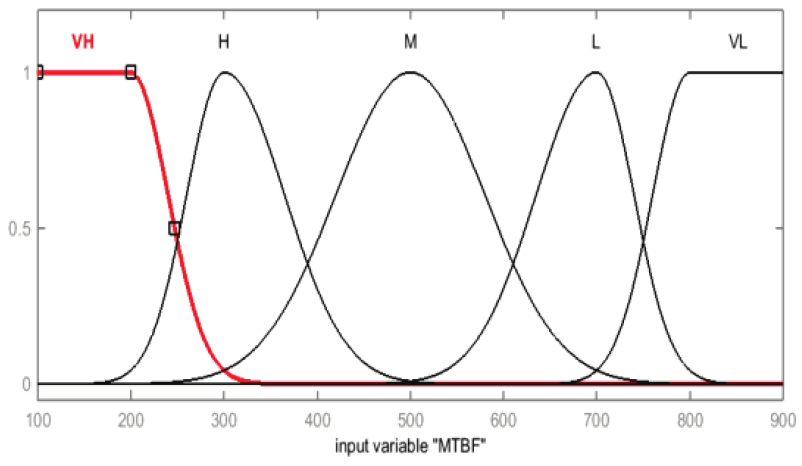

Fig. 5. GCMF of MTBF.

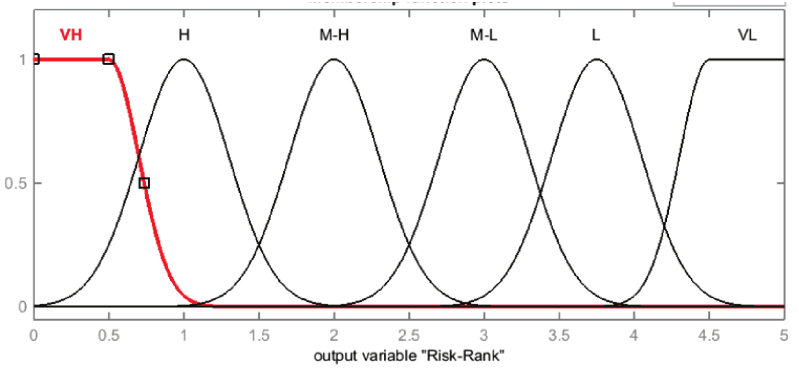

Fig. 6. GCMF of risk rank.

Figure 7 illustrates a rule view and an example calculation of a risk rank for machinery. The calculation was carried out for the PoNCP $=2.5$ and $\mathrm{MTBF}=500 \mathrm{hrs}$. The risk rank estimated by the fuzzy inference process is 1.97. The corresponding linguistic value is $\mathrm{M}-\mathrm{H}$ (using the membership function in Fig. 7). This linguistic value will be used for the classification of the machinery into groups for allocating maintenance resources (i.e. machinery with high risk will be given the main priority). Similarly, it is possible to calculate the risk of potential failures for each machinery.

Based on the manufacturing firm risk philosophy, it is possible to prioritise: 1. PS, 2. PoNCP and 3. A or any other sequence. This kind of prioritisation is mostly dependent on how the particular manufacturing firms perceive the risk in their operations related to health, safety, environment. and quality (i.e. HSE\&Q). 

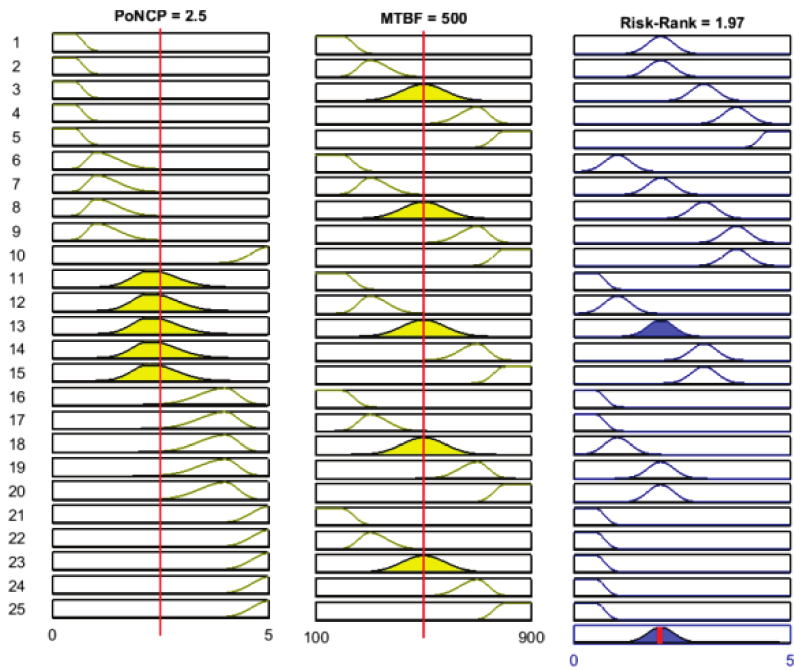

Fig. 7. Rule view and calculation [risk rank is 1.97 for $\mathrm{PoNCP}=2.5$ and $\mathrm{MTBF}=500]$.

Figure 8 illustrates a three-dimensional (3D) risk profile in relation to PoNCP and MTBF.

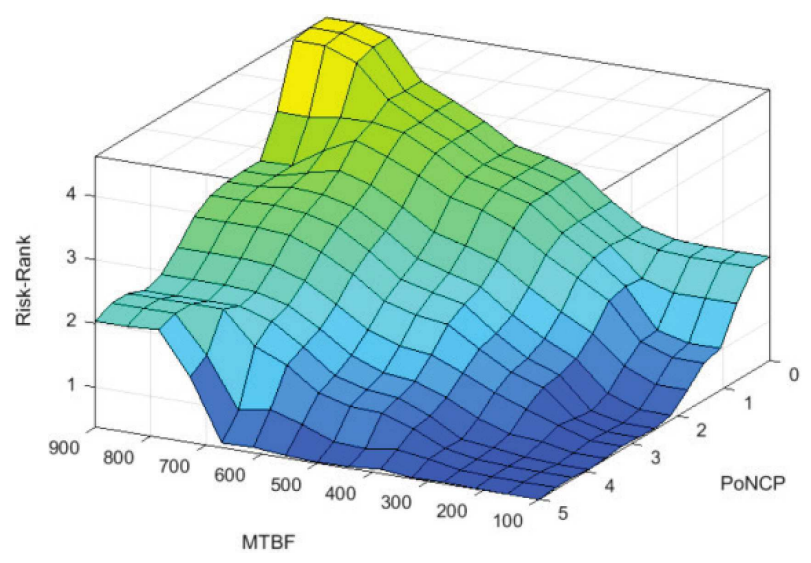

Fig. 8. 3D risk profile.

\section{Conclusions}

This work has demonstrated an RBM-related assessment and prioritisation approach for the machinery used in a manufacturing firm. It is proposed as an alternative to the currently existing empirical model-based approach, which poses significant challenges in allocating limited resources available in the studied manufacturing firm. A risk matrix was developed to overcome some of the existing challenges. A fuzzy logic-based modelling was used to minimise the suboptimal assessments and prioritisations. An illustrative fuzzy logic-based risk rank calculation approach was presented. The suggested RBM approach, together with a fuzzy inferencing process, enables to minimise suboptimal calculations when the input values are at the boundaries of the particular ranges. Fuzzy membership functions, together with the rule base, enable to insert the numbers with least uncertainty. When the membership functions are developed/revised with the support of maintenance experts, then, it also enables to recycle the experts' knowledge in the maintenance related decisions. Such recycling enables to minimise the variability in the final RBM prioritisations.

Future research will be carried out to develop the functions with the support of the data-driven modelling present in the artificial neural networks (ANN).

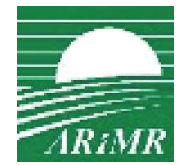

7th International Conference on Engineering, Project, and Production Management (EPPM2016) was co-organised by the Agency for Restructuring and Modernisation of Agriculture (Poland).

\section{References}

[1] Parida A., Kumar U., Maintenance productivity and performance measurement, Handbook of maintenance management and engineering, SpringerVerlag London Limited, part I, 17-41, 2009.

[2] Duffuaa S.O., Daya M.B., Turnaround maintenance in petrochemical industry: Practices and suggested improvements, J. Qual. in Maint. Eng., 10, 184-190, 2004.

[3] Daya M.B., Duffuaa S.O., Raouf A., Knezevic J., Ait-Kadi D., Handbook of maintenance management and engineering, Springer-Verlag London Limited, 2009.

[4] Daya M.B., Duffuaa S.O., Maintenance and Quality: The Missing Link, J. Qual. in Maint. Eng., 1, 20-26, 1995.

[5] Wenchi S., Wang J., Wang X., Chong H.Y., An application of value stream mapping for turnaround maintenance in oil and gas industry: Case study and lessions learned, in: Raidén A.B., Aboagye-Nimo E. [Eds.], Proc. 31st Annual ARCOM Conference, 7-9 September 2015, Association of Researchers in Construction Management, Lincoln, 813-822, 2015.

[6] Kurniati N., Yeh R.H., Lin J., Quality Inspection and Maintenance: The Framework of Interaction. Industrial Engineering and Service Science, IESS 2015 Proced. Manuf., 4, 244-251, 2015.

[7] Ratnayake R.M.C., Stadnicka D., Antosz K., Deriving an Empirical Model for Machinery Prioritization: Mechanical Systems Maintenance, Proceedings of the IEEE International Conference on Industrial 
Engineering and Engineering Management (IEEM), Bangkok, 1442-1447, 2013.

[8] Ratnayake R.M.C., Plant Systems and Equipment Maintenance: Use of Fuzzy Logic for Criticality Assessment in NORSOK Standard Z-008, Proceedings of the IEEE International Conference on Industrial Engineering and Engineering Management (IEEM), Bangkok, 1468-1472, 2013.

[9] Ratnayake R.M.C., KBE Development for Criticality Classification of Mechanical Equipment: A Fuzzy Expert System, Int. J. Dis. and Risk. Red., 9, 84-98, 2014.

[10] Piegat A., Modelling and fuzzy control [in Polish: Modelowanie i sterowanie rozmyte], Akademicka Oficyna Wydawnicza EXIT, Warszawa, 1999.

[11] Stadnicka D., Antosz K., Ratnayake R.M.C., Prioritization of Maintenance Tasks: Development of an Empirical Formula for Machine Classification, Saf. Sci., 63, 34-41, 2014.
[12] Tay K.M, Lim C.P., On the use of fuzzy inference techniques in assessment models: part II: industrial applications', Fuz. Opti. and Dec. Mak., 3, 283-302, 2008.

[13] Ratnayake R.M.C., Knowledge based engineering approach for subsea pipeline systems' FFR assessment: A fuzzy expert system, The TQM Journal, 28, 40-61, 2016.

[14] Hameed I.A., Using Gaussian membership functions for improving the reliability and robustness of students' evaluation systems, Expert Systems with Applications, 38, 6, 7135-7142, 2011.

[15] Mathworks, Fuzzy inference system modelling: Gaussian combination membership function, 2014, available: http://www.mathworks.se/help/fuzzy/ gauss2mf.html, last accessed 5th June 2016.

[16] Matlab, MATLAB 7.12.0 (R2014b), Fuzzy logic Toolbox, 1984-2014 The MathWorks, Inc., 2014. 